\title{
Inversor SPWM de alta eficiencia y baja distorsión armónica
}

Jairo Luis Gutiérrez Torres*

Miguel E. Ramírez C"**

Rodolfo Torres Castillo***

\section{Resumen}

Este documento presenta los resultados del diseño de un convertidor DC-AC con la técnica SPWM para obtener como salida una onda seno pura, después de un proceso de conversión energética con altos niveles de eficiencia y bajos niveles de distorsión armónica. El diseño electrónico seleccionado se orientó para lograr una implementación de bajo costo en sistemas fotovoltaicos para uso doméstico, lo que aporta, con una solución amigable con el medio ambiente, a la resolución del problema de cobertura energética que tienen las regiones apartadas de Colombia.

Palabras clave: armónicos, eficiencia, energía, inversor, potencia, señales

\begin{abstract}
This document presents the results of the design of a DC-AC converter with the SPWM technique, to obtain a pure sine wave as an output after an energy conversion process, with high levels of efficiency and low levels of harmonic distortion. The selected electronic design was oriented to achieve a low-cost implementation in photovoltaic systems for domestic use, providing an environmentally friendly solution to the problem of energy coverage in remote regions of Colombia.
\end{abstract}

Keywords: Efficiency, Energy, Harmonics, Inverter, Power, Signals

* Ingeniero electrónico especializado en Educación Superior a Distancia. Magíster en Ingeniería de Control y Automatización de Procesos. Contacto: jairol.gutierrez@unad.edu.co

** Estudiante del programa de Ingeniería Electrónica de la Universidad Nacional Abierta y a Distancia (UNAD). Adscrito al semillero de investigación BioVolta del grupo GIDESTEC de la Universidad Nacional abierta y a Distancia (UNAD). Contacto: migueleduar6@gmail.com

${ }^{* * *}$ Estudiante del programa de Ingeniería Electrónica de la Universidad Nacional Abierta y a Distancia (UNAD). Adscrito al semillero de investigación BioVolta del grupo GIDESTEC de la Universidad Nacional abierta y a Distancia (UNAD). Contacto: rtorrescastillo1@gmail.com 


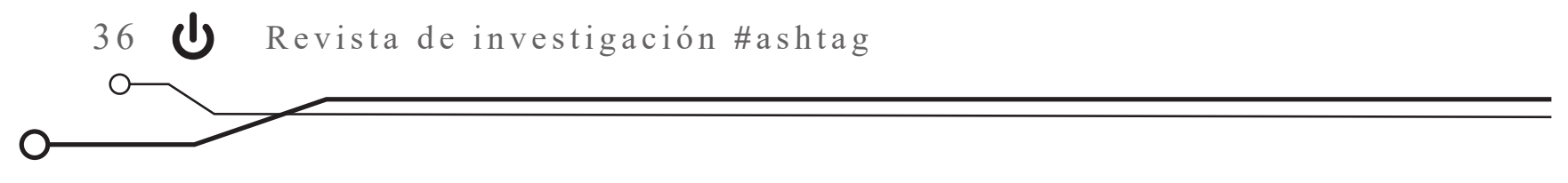

\section{Introducción}

La producción de energía a nivel mundial es directamente proporcional al daño al medio ambiente. Esto se explica por al uso excesivo e indiscriminado de combustibles fósiles en termoeléctricas y refinerías, así como por el uso de elementos radiactivos en la generación de energía nuclear; y el daño ecológico causado por represas en la generación de la energía hidroeléctrica., sumado al agotamiento de los combustibles fósiles e hídricos.

También debemos tener en cuenta la gran deficiencia en el suministro de energía eléctrica en muchos países, como es el caso de Colombia, en donde, aunque un gran número de regiones y comunidades se encuentran aisladas de la energía convencional, en otras existe un gran despilfarro de energía.

Es de gran relevancia, en el ámbito académico y científico, la búsqueda de la mayor eficiencia en el uso de la energía eléctrica, tanto a nivel

\section{Planteamiento del problema}

En los últimos años, se han propuesto y desarrollado sistemas alternativos para la generación de energía eléctrica en el mundo, dado el agotamiento, dependencia y constante incremento en el costo de los combustibles fósiles (carbón, petróleo y gas). El uso de estos combustibles, en -por ejemplo- termoeléctricas, provoca un enorme impacto negativo sobre el medio ambiente, en razón de los gases como el dióxido de azufre (SO2) y el dióxido de carbono (CO2) que se producen en su combustión. La emisión descontrolada de estos gases ha provocado daños medioambientales como el efecto invernadero, causante de la lluvia ácida y del calentamiento global. domiciliario como industrial, para reducir las malas prácticas en el uso de la energía eléctrica y darle paso a la aparición de nuevas técnicas de ahorro de energía y a la invención de nuevos dispositivos eléctricos para su ahorro, como lo son los controladores inteligentes, inversores, sistemas inverter y electrodomésticos eficientes.

Una buena alternativa para contrarrestar esta problemática es el gran desarrollo e impulso que han tenido las fuentes de energía alternativas, entre estas la energía fotovoltaica, eólica, y la mareomotriz, que aprovechan recursos naturales e inagotables como fuente energética.

Por otro lado, para la generación de energía no tradicional es necesario convertir la energía eléctrica (DC), obtenida ya sea de paneles o de aerogeneradores, en energía utilizable por los equipos eléctricos, o AC, algo que no sería posible sin la utilización de un inversor eficiente (Torres, Ramirez, y Gutierrez, 2016).

Los sistemas energéticos alternativos basados en energías renovables -como la energía solar, la eólica, la mareomotriz, biomasa, entre otrasson la respuesta a la crisis energética que afecta al planeta. De entre estas fuentes, se destaca que la energía solar y la eólica son las más limpias en su uso, pues no contaminan el medio ambiente.

En el mercado actual de los inversores, encontramos varias opciones, desde las más económicas hasta las más costosas, dependiendo de su eficiencia, características operativas, y de parámetros como la forma de onda, potencia de salida y técnica de control. 
En la actualidad encontramos inversores de onda cuadrada y onda modificada. Estos últimos son de bajo rendimiento dadas sus topologías y o características, que no son compatibles con cargas inductivas. También están disponibles lo inversores de onda seno pura, con técnica de control SPWM, que son aptos para uso domiciliario e industrial (Valencia, Saldarriaga y Giraldo, 2013).

En síntesis, los inversores de baja eficiencia producirán pérdidas de rendimiento y reducción de la vida útil de las cargas que le sean conectadas. Por ello, surge la necesidad de diseñar e implementar un inversor SPWM con onda de salida seno pura de alta eficiencia y bajo costo, para ser utilizado en sistemas energéticos alternativos de uso doméstico e industrial.

Los inversores con técnicas SPWM son altamente eficientes y de muy baja distorsión, lo que da como resultado la solución para la necesidad de la sociedad, las regiones y o comunidades alejadas, de energía convencional, pues se puede esta se puede utilizar en ambulancias e instrumentos médicos, así como en otros medios, con lo que se garantiza la vida útil en los electrodomésticos conectados a él.

\section{Marco teórico}

\section{Inversor}

Los inversores se ubican en la electrónica de potencia en el campo de la conversión energética, en concreto en la conversión continua-alterna (DC/AC). La evolución que han experimentado los semiconductores en términos de frecuencia de conmutación, pérdidas en conducción y facilidad de gobierno ha contribuido en gran medida a la popularización de este tipo de convertidores y de su evolución. En este tipo de equipos, de mediana-alta potencia, la tendencia es disminuir los costes y aumentar la eficiencia (González, 1999).

Los inversores son utilizados en una gran variedad de aplicaciones, desde pequeñas fuentes de alimentación ininterrumpidas (UPS) para computadoras, hasta aplicaciones industriales para manejar alta potencia. Los inversores también son utilizados para convertir la corriente continua generada por los paneles solares fotovoltaicos, acumuladores, baterías, etc., en corriente

alterna, y de esta manera poder ser inyectados en la red eléctrica, o usados en instalaciones eléctricas aisladas.

En un inversor se puede obtener un voltaje de salida variable si se varía así mismo la ganancia del inversor. Este proceso por lo general se hace al controlar la modulación del ancho de pulso (PWM) dentro del inversor. La ganancia del inversor se puede definir como la relación entre el voltaje de salida en AC y el voltaje de entrada en DC (Rashid, 2015).

La técnica de modulación SPWM unipolar es la más usada para la fabricación de inversores con onda de salida seno pura, por su ventaja sobre los armónicos, en cuanto a que estos últimos, en la salida, aparecen a mayor frecuencia. En ese sentido, la técnica SPWM facilita el filtrado y la reducción del tamaño de los componentes en el filtro (López, 2007). 


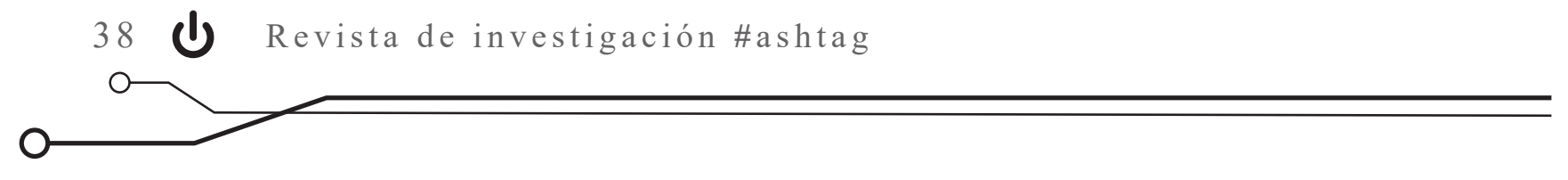

\section{Oscilador}

Un oscilador es un circuito que produce una oscilación propia de frecuencia, con forma de onda y amplitud determinadas. Todos los osciladores involucran uno o más elementos almacenadores

\section{Etapa driver y de potencia}

La etapa driver es la que gobierna la conmutación de los dispositivos, o interruptores de potencia, como MOSFET o IGBT. En este caso se han escogido los MOSFET, por sus características y ventajas frente a los IGBT. Como la señal de control SPWM generada en el circuito anterior es muy débil, es necesaria la implementación de una interfaz, o driver, que acondicione esta señal

\section{El transformador $y$ filtrado}

Un transformador eléctrico es una máquina estática de corriente alterna que permite variar alguna función de la corriente, como el voltaje o la intensidad, manteniendo la frecuencia y la potencia, en el caso de un transformador ideal. Para lograrlo, transforma la electricidad que le llega al devanado de entrada en magnetismo,

\section{Solución Propuesta}

Para generar el control de la conmutación por modulación SPWM, son necesarias dos formas de onda: una, llamada señal moduladora, es una onda senoidal; la segunda, llamada señal portadora, es una onda triangular. Por lo anterior se requiere la generación de una onda senoidal pura de frecuencia $60 \mathrm{~Hz}$. de energía, clasificables según su tipo. Tenemos, así, los osciladores LC, que utilizan capacitores e inductores; y los osciladores RC, que utilizan capacitores y resistores (Miyara, 2004).

correctamente para el accionamiento de los MOSFET (Moloney, 2006).

La siguiente etapa es la de potencia, que se construye con un puente $\mathrm{H}$, usando interruptores de estado sólido como MOSFET, IGBT, entre otros. De esta forma se puede crear una interfaz entre la fuente y la carga, a través del proceso de conversión DC-AC (Hart. 2001).

para volver a transformarla en electricidad, en las condiciones deseadas, en el devanado secundario (Alvares, 2009). La aplicación de un filtro para bajas responde a la atenuación de armónicos para obtener una tensión muy cercana a la sinusoidal pura (Xianjun, Lingshun, y Shutuan, 2005).

Se contemplaron dos alternativas para la generación de la onda senoidal: usar componentes análogos, implementando un circuito oscilador senoidal; o hacer uso de un microcontrolador. 
Figura 1. Oscilador Bubba en Multisim.

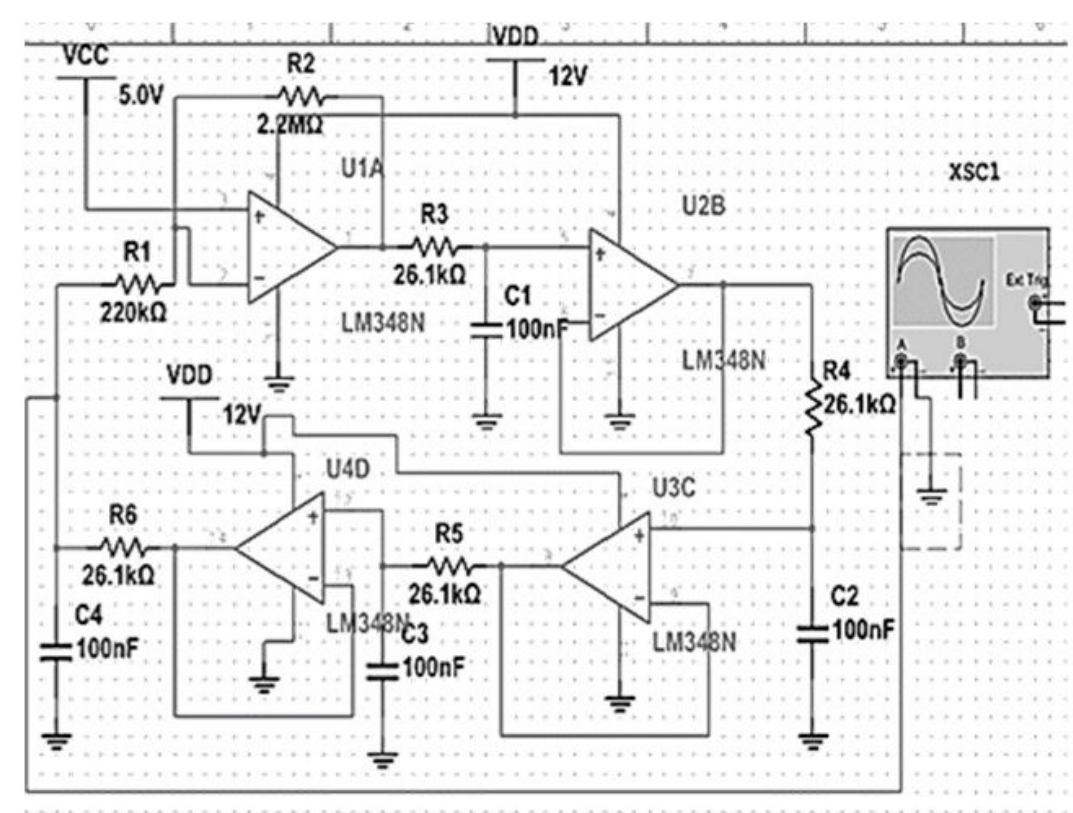

Fuente: elaboración propia.

Inicialmente en el desarrollo de la investigación, se trabajó el diseño de un oscilador analógico $\mathrm{RC}$, pero se seleccionó el Bubba, que es un oscilador por desplazamiento de fase, que entrega una señal senoidal pura de baja distorsión armónica y muy estable en frecuencia. Los cálculos necesarios para encontrar la frecuencia de salida de $60 \mathrm{~Hz}$ se muestran a continuación.

\section{Oscilador de onda triangular}

El siguiente paso en el diseño es producir una onda triangular a través de medios analógicos, para ser comparada con la sinusoidal, obtenida del oscilador.

Para esto, se diseñó un oscilador de onda cuadrada y se inyectó a un circuito integrador, y así obtener la señal triangular requerida. Se seleccionó el oscilador por relajación. $f=1 /(2 \pi R * C)$

(Ecuación 1)

Donde: $\mathrm{f}=$ frecuencia; $\mathrm{R}=$ Resistencia; $\mathrm{C}=$ Capacitor.

La fórmula para calcular la frecuencia de oscilación es:

$$
\begin{aligned}
& f=1 / 2 R C \\
& \text { (Ecuación 2) }
\end{aligned}
$$

Donde: $\mathrm{f}=$ frecuencia; $\mathrm{R}=$ Resistencia; C= Capacitor. Siendo R2 0,86 veces mayor a R1. 


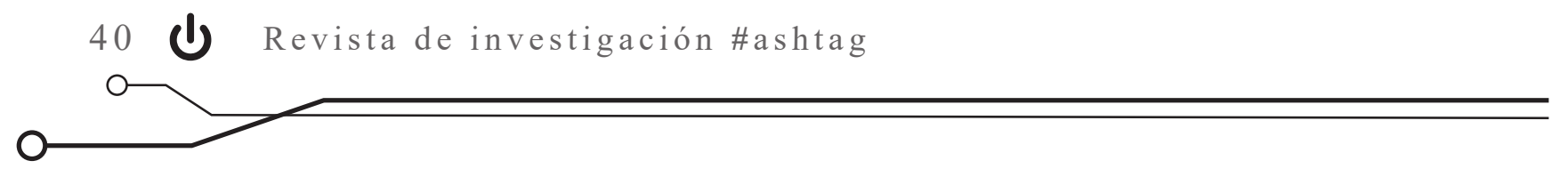

\section{Circuito integrador de señal}

El siguiente circuito es el integrador de señales. Este permite integrar las señales que se le suministran en su entrada, por medio de condensadores, resistencias y AO conectados en paralelo, a través del bucle de retroalimentación negativa. Como es sabido que la integral de una señal cuadrada es una triangular, la salida de este circuito entregará a su salida una señal triangular, con

\section{Índice de modulación en amplitud}

$$
\mathrm{ma}=\frac{V \operatorname{sen} P}{V \operatorname{tri} P}
$$

(Ecuación 3)

Donde: ma=Indice de amplitud; VsenP=amplitud de la señal senoidal; VtriP=Amplitud de la señal portadora.

\section{Índice de modulación en frecuencia}

El índice de modulación en frecuencia $(m f)$ es la relación entre la frecuencia de la señal portadora y la frecuencia de la señal moduladora. A continuación, se $m f$. Si $m f<21$ se dice que un inversor está muy poco modulado, mientras que si $m f>$ 21 se dice que está muy modulado.

$$
m_{f}=\frac{f_{\text {tri }}}{f_{\text {sen }}}
$$

(Ecuación 4) igual frecuencia de la señal cuadrada de entrada, ya que esta no la modifica en ningún momento.

Aunque las señales obtenidas con circuitos analógicos, son muy precisas y estables. Al utilizar circuitos digitales, se pueden obtener mejores resultados, sumados a que estos son menos robustos y en sistemas de control son más eficientes.

En relación con el índice de modulación en amplitud ma, para una topología de puente completo, según el valor de ma, se pueden distinguir tres zonas de modulación. Una de ellas sería $0<$ $m a<1$ zona lineal, en la que el ancho de los pulsos aumenta a medida que lo hace la tensión de referencia; otra, $1<$ ma $<3,54$ sobre modulación, en la que dos o más pulsos se unen formando un único pulso; y ma $>3,54$ Onda cuadrada en la que únicamente hay un pulso por semiciclo.

Donde $\mathrm{m}_{\mathrm{f}}=$ modulacion de frecuencia; $\mathrm{f}_{\text {tri }}=$ frecuencia portadora; $f_{\text {sen }}=$ frecuencia senoidal.

Aquí se integran las señales obtenidas por medio de los circuitos anteriormente mencionados, para ser comparadas y generar la señal spwM. Se necesita de esta señal para controlar el disparo de los MOSFET. 
Figura 2. Señales SPWM unipolar.
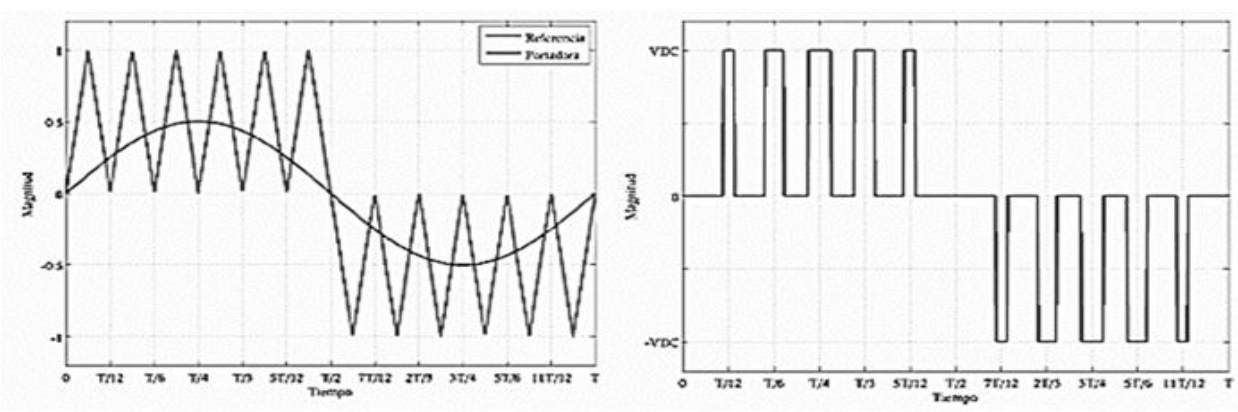

Fuente: Elaboración propia.

Como se mencionó anteriormente, estas señales, en comparación con las bipolares, son más adecuadas para el control de puentes $\mathrm{H}$, ya

\section{Etapa driver}

En este caso, el disparo de los dispositivos interruptores de potencia se contempló a través de los drivers de la familia IR21xx. Estos circuitos integrados permiten acondicionar la señal SPWM de control que llega a las compuertas de los MOSFET para su accionamiento. Los drivers poseen dos entradas para recibir las señales SPWM, una para recibir la señal alta y otra para recibir su complementada, lo que le permite el control de dos MOSFET, al mismo tiempo o de una rama de un puente $\mathrm{H}$. Es por esto que se necesitan dos CI IRF210 para controlar un puente H completo. que permiten un mayor aprovechamiento en el manejo de la potencia eléctrica de salida en el inversor.
Este circuito tiene la ventaja de generar un tiempo muerto entre sus señales SPWM de salida, lo que evita posibles daños ocasionados por cortocircuitos, que generalmente se presentan por accionamiento de los interruptores de una sola rama al mismo tiempo.

A continuación, se muestra la imagen de un CI IR2110, controlando medio puente H. Para el caso de un puente $\mathrm{H}$ Completo sería necesario utilizar dos CI IR2110.

Figura 3. Driver para medio puente $\mathrm{H}$

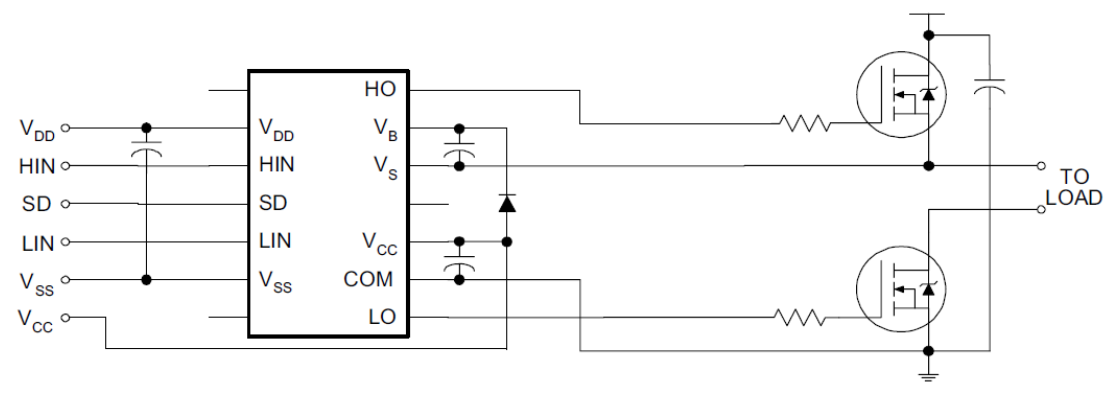




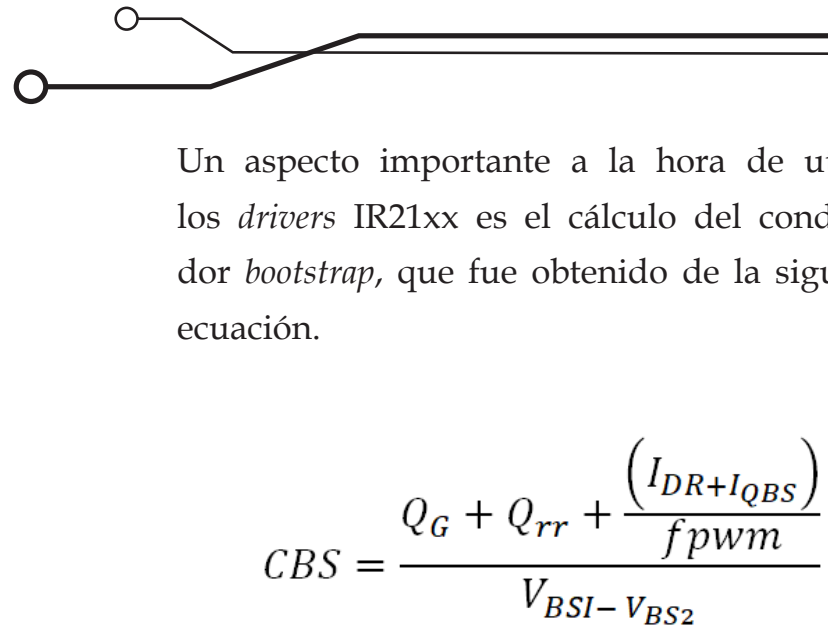

(Ecuación 5)

El condensador bootstrap es utilizado para generar una fuente flotante en el driver. Este hace que las compuertas de los MOSFET reciban una señal de voltaje adecuada para su funcionamiento. Esto es posible gracias al establecimiento de dos puntos de referencia de voltaje, uno en la parte baja del puente $\mathrm{H}$, la cual es la tierra del circuito, y otro en la parte alta, o mitad del puente. Este condensador trabaja en carga y descarga a través de un diodo.

\section{Implementar un puente н para la conexión de los MOSFET}

El puente H que se ha diseñado es apto para trabajar con una fuente de voltaje de 12 a 48 vDC (ver figura 4), por lo que los MOSFET escogidos para esta configuración deberán soportar un gran amperaje, ya que deben entregar a su salida una potencia máxima de 900 watts. Además deben ser semiconductores muy eficientes, que consuman poca potencia durante su funcionamiento, y posean una velocidad de conmutación muy elevada. Las características de robustez y eficiencia de estos interruptores son satisfechas totalmente por los MOSFET de potencia, fácilmente adquiribles en el mercado. Los MOSFET utilizados en este circuito fueron el IRP2990, IRF3550, IRF3205, entre otros.

Figura 4. Puente $\mathrm{H}$

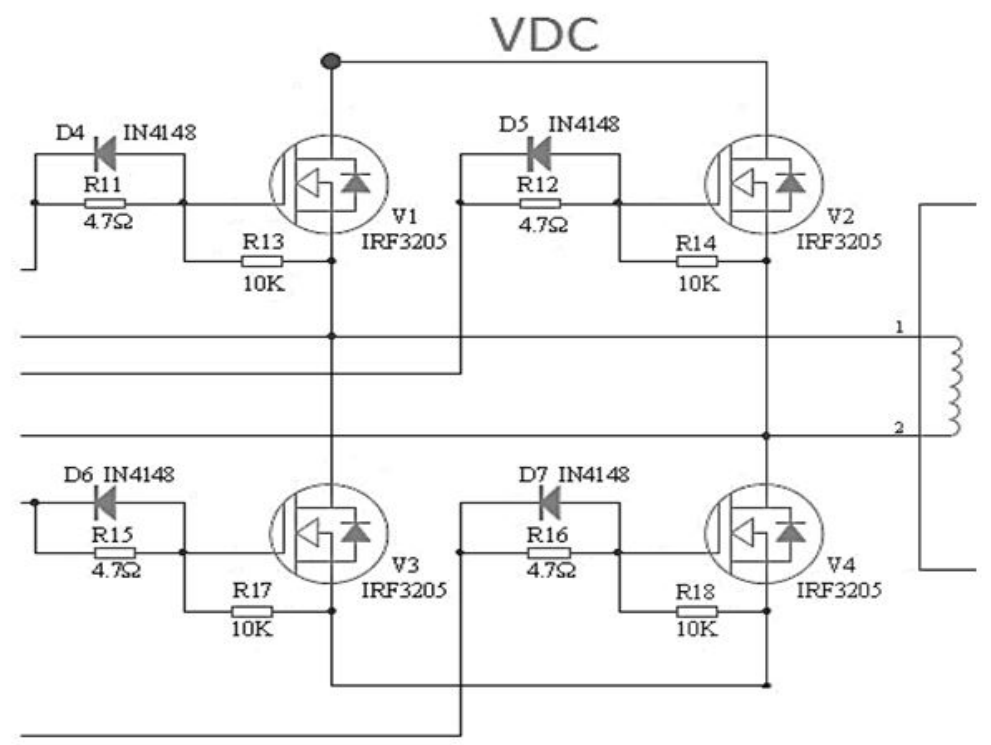

Fuente: Microelectronics Corporation, EG. (2014) 


\section{Elevación y filtrado}

En este diseño se propuso la implementación de un transformador elevador toroidal, en el que el devanado primario opera a 16 voltios AC y el secundario a 120 AC. Debido a que la señal que sale del puente $\mathrm{H}$ no es sinusoidal, sino más bien una serie de pulso cuadrados con una frecuencia de 23,5 Hz que es la frecuencia de la portadora, se hace necesario la implementación de un filtro pasa bajas que deje pasar solo el armónico fundamental de $60 \mathrm{~Hz}$, que es el ideal para el buen funcionamiento de electrodomésticos y dispositivos eléctricos y electrónicos.
A continuación se muestra el diseño del filtro pasa bajas para el circuito inversor. Un filtro pasa baja LC serie es un circuito formado por una bobina y un condensador en serie, en el que la salida de la señal filtrada se toma en los extremos del condensador (Delgado, 2012), como se muestra en la (figura 5). Y cuyo análisis se puede hacer con la siguiente ecuación que describe su frecuencia de resonancia.

Figura 5. Filtro LC calculado

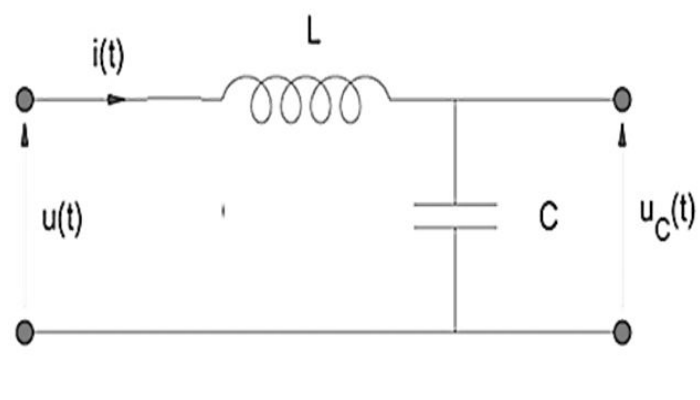

Fuente: elaboración propia.

$$
f r=\frac{1}{2 \pi * \sqrt{L C}}
$$

(Ecuación 6)

Donde: fr es frecuencia de cort; $L=$ es inductancia; $C=$ es capacitancia

\section{Resultados}

- Se diseñó un oscilador que entrega una frecuencia de $60 \mathrm{~Hz}$ muy estable.

- Se implementó un oscilador de onda triangular para generar la señal portadora.
- Se diseñó un circuito modulador sPwM.

- Se calculó del condensador bootstrap de la etapa driver. 


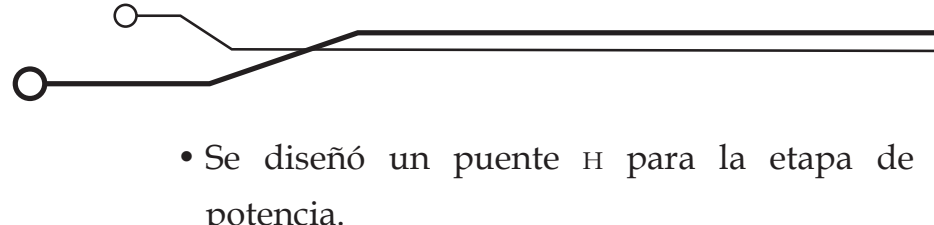

- Se calculó el bobinado del transformador elevador final para la carga

- Se diseña un filtro pasivo pasa bajas LC supresor de distorsión armónica.
En el desarrollo de este proyecto, se obtuvieron los resultados esperados y calculados, y al realizar las debidas simulaciones. En la siguiente figura se presentan las señales obtenidas en la modulación y la salida sinusoidal alimentando una carga no lineal.

Figura 6. Señal modulada y filtrada SPWM

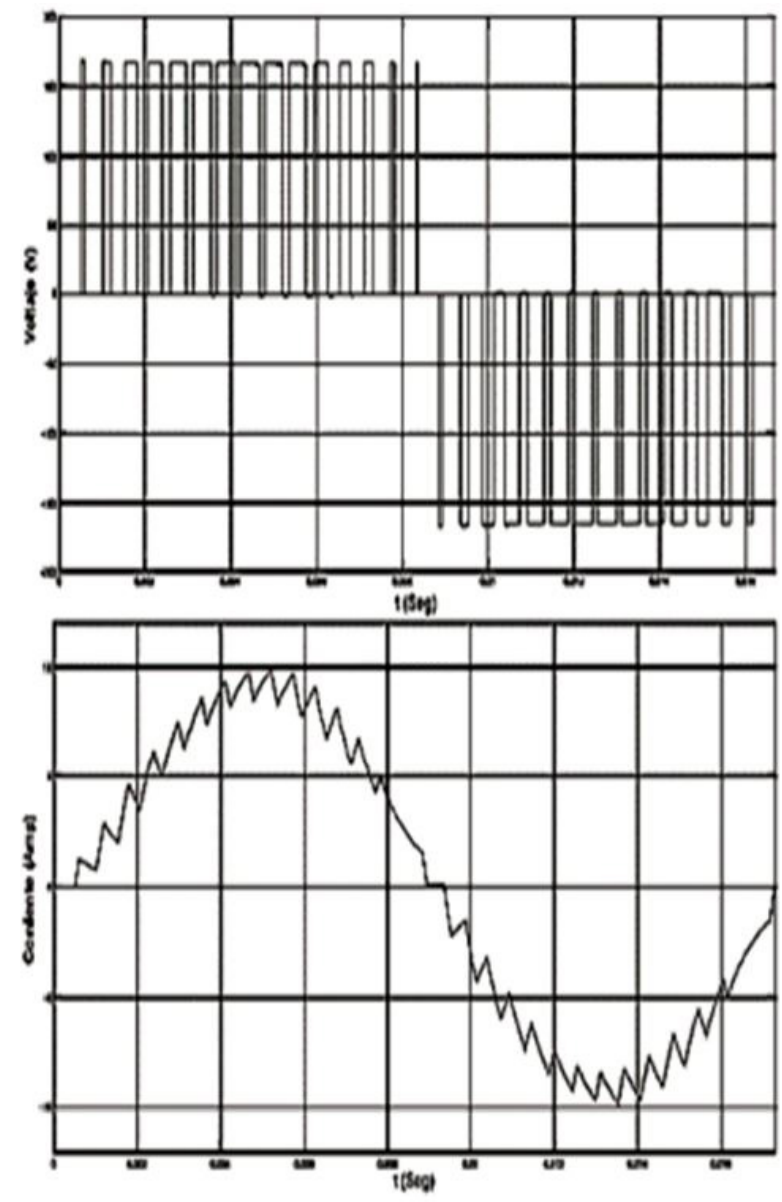

Fuente: elaboración propia.

\section{Conclusiones}

En la elaboración del diseño del inversor SPWM, se logró obtener resultados satisfactorios referentes a la aplicación de electrónica discreta para el proceso de conversión de energía DC-AC con un alto nivel de eficiencia: al rededor del $96 \%$ con carga resistiva y un $90 \%$ en cargas reactivas. 
Se logró la apropiación de habilidades y destrezas en electrónica de potencia área, que es la vanguardia del desarrollo productivo global, en aras de continuar investigaciones relacionadas con la mejora de las condiciones de generación de energía en el desarrollo productivo nacional, con soluciones amigables con el medio ambiente.
La implementación de convertidores DC-AC en el uso de las fuentes no convencionales de energía a un bajo costo aportará a las comunidades una solución a la necesidad de obtener energía en lugares sin energía convencional. También contribuye a, que la industria tome el timón de las buenas prácticas de las energías renovables, y así mitigar la contaminación ambiental, con un aporte a la resolución de la crisis energética actual.

\section{Referencias}

Delgado, M. (2012). Diseño e implementación experimental de un inversor monofásico operando en modo isla (trabajo de grado). Universidad Carlos III, Madrid, España. Recuperado de https:// core.ac.uk/download/pdf/29403356.pdf

Gonzalez, J. (1999). Inversores PWM [documento interno de trabajo]. Gijón: Universidad de Oviedo. Recuperado de: http://www.iee-pels-ies.es/Pels/Pdf/Leccion\%20Inversores\%20Juan \%20Diaz.pdf

Hart, D. (2001) Electrónica de potencia. Madrid: Prentice Hall.

International Rectifier, IR. (2005). IR2110(-1-2)(S)PbF / IR2113(-1-2)(S)PbF High and Low Side Driver. Data Sheet No. PD60147 rev. U. Recuperado de https://pdf1.alldatasheet.com/ datasheet-pdf/view/82793/IRF/IR2110.html

López, D. J., Camacho, G. A., Díaz J. O., y Gaviria, C. A. (2007). Modulación pwm aplicada a inversores trifásicos dentro del esquema de accionamientos eléctricos AC [versión preliminar de artículo sin publicar]. Popayán: Universidad del Cauca. Recuperado de https://www.academia. edu/28030774/MODULACI\%C3\%93N_PWM_APLICADA_A_INVERSORES_TRIF\%C3\%81SICOS_DENTRO_DEL_ESQUEMA_DE_ACCIONAMIENTOS_EL\%C3\%89CTRICOS_AC

Microelectronics Corporation, EG. (2014). EG8010 Datasheets - Single Phase Sinusoid Inverter ASIC. Recuperado de https://www.lz2gl.com/data/power-inverter-3kw/eg8010_ datasheet_en.pdf

Miyara, F. (2004). Osciladores sinusoidales. Departamento de Electrónica, Universidad Nacional de Rosario, Argentina. Recuperado de https://catedra.ing.unlp.edu.ar/electrotecnia/electronicos2/download/Apuntes/oscilad\%20sinus\%20MIYARA.pdf 


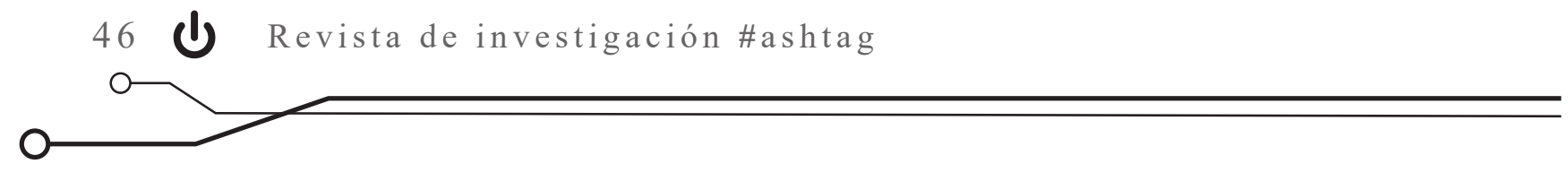

Moloney, T. (2006). Electrónica industrial moderna. Ciudad de México: Pearson Educación.

Rashid, M. (2015 cuarta edición). Electrónica de potencia. Ciudad de México: Prentice Hall Hispanoamericana.

Torres, R., Ramirez, M. y Gutierrez, J. (2016). Diseño de un inversor SPWM de onda seno pura de alta eficiencia. Riohacha: UNAD

Valencia, H., Saldarriaga, M. y Giraldo, J. (2013). Fundamentos de electrónica industrial. Medellín: Universidad Pontificia Bolivariana.

Redondo, F. y Redondo R. (2005). Redes eléctricas de Kirchhoff con 400 problemas resueltos. Salamanca: Revides. Recuperado de https://www.worldcat.org/title/redeselectricas-de-kirchhoff-con-400-problemas-resueltos/oclc/432962522?referer $=$ di\&ht $=$ edition 